\title{
OCORRÊNCIA FÚNGICA EM ACERVOS BIBLIOGRÁFICOS DO MUNICÍPIO DE FORTALEZA, CEARÁ, BRASIL
}

FUNGAL OCCURRENCE IN LIBRARY COLLECTIONS IN THE CITY OF FORTALEZA, CEARÁ, BRAZIL

\section{APARICIÓN DE HONGOS EN LAS COLECCIONES DE LA BIBLIOTECA} EN LA CIUDAD DE FORTALEZA, CEARÁ, BRASIL

\author{
Germana Costa Paixão ${ }^{1}$ \\ Roberta Silva Rizzo ${ }^{2}$ \\ Ari Clecius Alves de Lima ${ }^{3}$ \\ Lydia Dayanne Maia Pantoja ${ }^{4}$
}

\section{RESUMO}

$\mathrm{Na}$ atualidade a conservação dos documentos bibliográficos ainda apresenta problemas complexos e diversificados, apesar da maior consciência do tema nos últimos anos. Nesse contexto, a presente pesquisa objetiva conhecer o espectro fúngico de acervos de bibliotecas públicas de referência no município de FortalezaCE. Bimestralmente, durante 24 meses, 05 amostras/biblioteca provenientes de livros e/ou documentos históricos escolhidos aleatoriamente foram analisadas, perfazendo no total 180 amostras. As amostras foram colhidas através de swabs estéreis, friccionados por 30 segundos e em seguida transportados em solução salina estéril. Ao chegarem ao laboratório, as amostras foram semeadas em meio de cultura Ágar Batata Dextrose (Himedia $\left.{ }^{\circledR}\right)$, incubadas por 7 dias $\left(26-28{ }^{\circ} \mathrm{C}\right)$ com posterior contagem global e identificação das colônias fúngicas baseada nas análises macro e micromorfológicas. Do total de obras analisadas $86 \%$ mostraram positividade para fungos. Nas bibliotecas A, B e C foram contabilizadas 3.612, 4.235 e 2.978 colônias fúngicas, respectivamente e identificados 55 diferentes achados fúngicos (29 gêneros e 26 espécies fúngicas), com destaque para os hialohifomicetos Aspergillus e Penicillium. Os dados revelaram alguns agrupamentos com forte correlação, isto é, quando um dos achados fúngicos ocorre, os demais achados do agrupamento também podem estar presentes nos livros analisados, a saber: Bibliotecas A e B (Aspergillus sp., Aspergillus flavus, Apergillus niger e Penicillium sp.) e Biblioteca C (Aspergillus niger, Trichoderma sp. e Penicillium sp.). A pesquisa permitiu oferecer aos gestores das bibliotecas, elementos para aprofundar seus conhecimentos na área de preservação e conservação de obras literárias de inestimável valor.

PALAVRAS-CHAVE: Fungo. Documentos bibliográficos. Biblioteca.

\section{ABSTRACT}

At present the conservation of bibliographic documents presents complex and diverse problems, despite greater awareness theme in recent years. In this context, this research aims to know the fungal spectrum of public reference library collections in the city of Fortaleza-CE. Every two months, 24 months, 05 samples / from

\footnotetext{
${ }^{1}$ Professora de Microbiologia. Curso de Ciências Biológicas da Universidade Estadual do Ceará. E-mail: germana.paixao@uece.br

${ }^{2}$ Graduanda em Farmácia pela Faculdade Cathedral (RR), exercendo atualmente as atividades profissionais no Hemocentro da Secretaria de Estado da Saúde de Roraima. E-mail: robertasilvarizzo@gmail.com

${ }^{3}$ Doutorado em Engenharia Civil (Recursos Hídricos) pela Universidade Federal do Ceará (2015). E-mail: ari072000@yahoo.com.br

${ }^{4}$ Doutoranda em Engenharia Civil (Saneamento Ambiental) pela Universidade Federal do Ceará. E-mail: lydiapantoja@yahoo.com.br
}

Enviado em: 20/09/2015 - Aceito em: 09/12/2015 
library books and / or randomly chosen historical documents have been analyzed, totaling 180 samples in total. Samples were harvested through sterile swabs, rubbed for 30 seconds and then transported in sterile saline. Upon arrival at the laboratory, samples were seeded in culture medium Potato Dextrose Agar (Himediaß) and incubated for 7 days $\left(26-28^{\circ} \mathrm{C}\right)$ with subsequent global count and identification of fungal colonies based on macro- and micromorphological. Of total works analyzed $86 \%$ were positive for fungi. In libraries A, B and C were accounted for 3,612, 4,235 and 2,978 fungal colonies, respectively and identified 55 different fungal findings (29 genera and 26 fungal species), highlighting the hialomicetos Aspergillus and Penicillium. The results showed a strong correlation with some clusters, that is, when a fungal findings occurs, the other group findings can also be present in the analyzed books, namely: Libraries A and B (Aspergillus sp, Aspergillus flavus, and Apergillus niger and Penicillium sp.) and C Library (Aspergillus niger, Trichoderma sp. and Penicillium sp.). The research allowed to offer managers of libraries, elements to deepen their knowledge in the field of preservation and conservation of literary invaluable.

KEYWORDS: Fungi. Bibliographic documents. Library.

\section{RESUMEN}

En la actualidad la conservación de los documentos bibliográficos presenta problemas complejos y diversos, a pesar de una mayor tema de la conciencia en los últimos años. En este contexto, esta investigación tiene como objetivo conocer el espectro de hongos de las colecciones de la biblioteca de referencia pública en la ciudad de Fortaleza-CE. Cada dos meses, 24 meses, 05 muestras / de libros de la biblioteca y / o documentos históricos elegidos al azar han sido analizados, un total de 180 muestras en total. Las muestras se recogieron a través de torundas estériles, se frotaban durante 30 segundos y luego transportados en solución salina estéril. A su llegada al laboratorio, las muestras se sembraron en medio de cultivo Agar de dextrosa de patata (Himediaß) y se incubaron durante 7 días $\left(26-28^{\circ} \mathrm{C}\right)$ con recuento mundial posterior y la identificación de las colonias de hongos basados en macro y micromorfológico. De las obras totales analizaron $86 \%$ fueron positivos para los hongos. En las bibliotecas A, B y C se contabilizaron 3.612, 4.235 y 2.978 colonias de hongos, respectivamente y se identificaron 55 resultados diferentes hongos (29 géneros y 26 especies de hongos), destacando los hialomicetos Aspergillus y Penicillium. Los resultados mostraron una fuerte correlación con algunos grupos, es decir, cuando se produce un hallazgos de hongos, los otros resultados de los grupos también pueden estar presentes en los libros analizados, a saber: Bibliotecas A y B (Aspergillus sp, Aspergillus flavus y Aspergillus niger y Penicillium sp.) y C Library (Aspergillus niger, Trichoderma sp. y Penicillium sp.). La investigación permitió ofrecer a los directivos de las bibliotecas, los elementos para profundizar sus conocimientos en el campo de la preservación y conservación de valor incalculable literaria.

PALABRAS CLAVE: Hongos. Documentos bibliográficos. Biblioteca.

\section{INTRODUÇÃO}

$\mathrm{Na}$ atualidade a conservação dos documentos bibliográficos ainda apresenta problemas complexos e diversificados, apesar da maior consciência para o tema nos últimos anos. O empirismo está cedendo espaço a processos técnico-científicos que privilegiam a conservação preventiva, apontando-a como necessária, eficaz e mais econômica que a conservação corretiva ou de restauração do objeto degradado. Não obstante, a conservação preventiva exige elevado grau de conhecimento, tanto do objeto como dos fenômenos e variáveis envolvidas (BARROS, 2009; PIACENTI, 2014).

Um dos principais componentes dos acervos bibliográficos ainda é o livro impresso, que por sua vez é constituído de elementos como o papel, o qual é sensível a agentes deteriorantes tais como umidade, pragas, calor, luminosidade e processos químicos como a acidez (CORADI; EGGERT-STEINDEL, 2008). Nesse sentido, torna-se fundamental que todos os que manipulam acervos preocupem-se em investigar quais fatores ambientais afetam a durabilidade e permanência do papel e delinear alguns conceitos informativos que se desenvolveram para prolongar a vida e utilidade das coleções bibliográficas. 
Dentre os vários agentes deteriorantes do papel, os biológicos se destacam como os mais danosos e, muitas vezes, irreparáveis. Dentre eles estão os insetos; os microorganismos; os roedores e o próprio ser humano (CORADI; EGGERT-STEINDEL, 2008).

No tocante aos diversos micro-organismos, os fungos podem agir como importantes biopoluentes de bibliotecas (PANTOJA et al., 2012), já que estas apresentam grandes volumes de substratos adequados à sua nutrição, sob a forma de papéis, tecidos, cola, pó e ar refrigerado.

Os fungos possuem nutrição do tipo quimio-heterótrofica e requerem a presença de uma fonte de carbono pré-formada em seu substrato. São capazes de metabolizar carboidratos complexos, necessitam de menos nitrogênio para um crescimento equivalente ao de bactérias e a maioria pode crescer em altas concentrações de açúcar ou sal, em suma, estão adaptados a uma alimentação a base de celulose (celulóticos), proteínas (proteolíticos) e amido (amilóticos) (MADIGAN; MARTINKO; PARKERT, 2010).

Os acervos das bibliotecas do Estado do Ceará são representados especialmente por livros e documentos em papel, seguido por obras confeccionadas com fibras de madeira, linho e algodão. Porém, independentemente do tipo de fibra constituinte, todo o papel possui em comum, o seu caráter higroscópico, ou seja, toda a fibra de papel absorve água e perde água de acordo com a taxa de umidade existente no local em que está sendo mantido (MÁRSICO, 1998; PIACENTI, 2014).

A relação entre os fungos e a umidade do ar é claramente estabelecida. Sabe-se que os fungos em sua fase de dormência não causam grandes danos aos livros, porém, quando ativos, por exemplo, em decorrência das elevadas taxas de umidade do ambiente, afetam diretamente os livros causando manchas, deixando-os frágeis e facilmente danificáveis (OGDEN, 2001; ARABIDIAN; SAAD, 2014). Isso ocorre em virtude da ação de enzimas extracelulares oxidativas secretadas pelos fungos, denominadas oxidoredutases, que degradam a celulose, que se caracteriza quimicamente como um polímero de celobiose (Dglucopyranosyl- $\beta$-1,4-D-glucopyranose) (MARTÍNEZ et al., 2005).

Os fungos Deuteromycetes tais como os gêneros Aspergillus, Penicillium, Trichoderma, Stachybooys, Stemphylium, Alternaria, Myrothecium seguidos pelos Zygomycetes (Rhizopus e Mucor) e Ascomycetes (Chaetomium) costumam ser os representantes fúngicos mais frequentemente associados a livros e documentos (GALLO, 1993; ROSA et al., 2008), podendo ainda ser isolados de adesivos, filmes plásticos, cera, selos, fitas magnéticas e microfilmes.

O conhecimento e o monitoramento da microflora presente em livros e documentos guardados em bibliotecas públicas objetivam prioritariamente impedir ou limitar os processos de degradação sobre os mesmos, contribuindo para a preservação do conhecimento escrito, garantindo sua disseminação por várias gerações e a um maior e sempre crescente número de pessoas. Adicionalmente, outros aspectos relacionados à presença de fungos nesses locais revestem-se de igual importância, merecendo destaque o potencial que esses micro- 
organismos possuem de atuarem como agentes desencadeadores de quadros de hipersensibilidades imediatas, mediante sua ação como aeroalérgenos (LI; KUO, 1992).

Nesse ínterim, a presente pesquisa objetiva conhecer o espectro fúngico de acervos de bibliotecas públicas e gerar aos gestores, elementos para aprofundar seus conhecimentos na área de preservação e conservação às obras literárias de inestimável valor.

\section{MÉTODO}

Foram escolhidas 03 bibliotecas públicas de referência no município de Fortaleza-CE, denominadas de Biblioteca A, Biblioteca B e Biblioteca C.

As bibliotecas foram selecionadas por apresentarem as seguintes peculiaridades: biblioteca A, fundada em 1880, é constituída de aproximadamente 120.000 livros de registros, classificadores, documentos históricos e revistas, distribuídos em $1.800 \mathrm{~m}^{2}$; a biblioteca $\mathrm{B}$ ocupa área de $200 \mathrm{~m}^{2}$, em prédio composto por dois andares onde se encontra distribuído o acervo de aproximadamente 15.000 títulos, voltados para os estudos de línguas e filosofia, e a biblioteca C, fundada em 1867, ocupa uma área geográfica de $2.272 \mathrm{~m}^{2}$, distribuídos em cinco pavimentos, com acervo de aproximadamente 100.000 volumes, incluindo obras raras nacionais. Durante 24 meses, bimestralmente, foram analisados 05 livros e/ou revistas científicas e/ou documentos históricos escolhidos de forma aleatória de cada biblioteca, perfazendo 180 amostras biológicas.

As amostras foram colhidas por meio da fricção de swabs estéreis diretamente na obra por 30 segundos, realizando-se movimentos em ziguezague por toda a página para maior cobertura do espaço de coleta . Os locais de fricção foram a primeira página interna, no centro da obra e na última página interna. Após a coleta, os swabs foram agitados em solução salina estéril a 0,85\% por 3 a 5 minutos (GAMBALE et al, 1993) e transportados ao Laboratório de Microbiologia (LAMIC) do Curso de Ciências Biológicas da Universidade Estadual do Ceará.

Ao chegarem ao laboratório, as amostras foram semeadas em placas de Petri contendo Ágar Batata Dextrose (Himedia $\left.{ }^{\circledR}\right)$ e incubadas à temperatura de $26-28{ }^{\circ} \mathrm{C}$ durante sete dias, realizando-se observações diárias. A partir do aparecimento visual de colônias fúngicas procedeu-se a contagem global das mesmas, categorizando-se por obra analisada. Por fim, a identificação das colônias fúngicas ocorreu com base em análises macro e micromorfológicas (HOOG; GUARRO; GENÉ, 2000; KONEMAN et al., 2001; SIDRIM; ROCHA, 2004).

Os dados catalogados permitiram a construção de dendogramas. Os itens com maior correlação foram agrupados considerando a distância de conexão completa (VALENTIN, 2000; HUSSON et al., 2009; R CORE TEAM, 2012). 


\section{RESULTADOS}

Das 180 obras analisadas $86 \%$ mostraram positividade para fungos. A contagem global de colônias de todas as obras das bibliotecas A, B e C resultou em 3.612, 4.235 e 2.978 colônias fúngicas, respectivamente. As obras foram catalogadas em três períodos de acordo com o ano de publicação (antes de 1800, entre 1801 a 1900 e depois de 1900). O número de colônias de cada uma das obras por período foi analisado e não mostraram relevância estatística $(\mathrm{p} \geq 0,05)$.

A alta contagem de unidades formadoras de colônias era esperada, devido principalmente à grande quantidade de substratos favoráveis à ação de biodegradadores/biopoluentes sobre os acervos escritos e digitais. Os dados corroboram com o estudo de Bortoletto e colaboradores, que, em 2002, constataram séria contaminação fúngica no ar do acervo da biblioteca de Manguinhos da Fundação Oswaldo Cruz, que à época contava com 620.000 volumes e provocou a interdição da biblioteca por 5 meses.

Foram identificados 55 grupos fúngicos, distribuídos em 29 gêneros e 26 espécies, com destaque para os gêneros de hialohifomicetos Aspergillus sp. (MICHELI, 1729) e Penicillium sp. (MICHELI, 1729), encontrados nas três bibliotecas. Os demais gêneros e espécies foram observados com menor frequência conforme demonstrado na tabela 1 . Os hifomicetos não identificados até gênero foram incluídos na ordem Agonomycetales (Mycelia sterilia). A composição do espectro de fungos foi formada predominantemente por deuteromicetos filamentosos hialinos.

TABELA 1 - Participação relativa (\%) dos principais isolados fúngicos nos acervos das bibliotecas A, B e C.

\begin{tabular}{|c|c|c|c|c|}
\hline \multicolumn{2}{|c|}{ Isolados Fúngicos } & \multicolumn{3}{|c|}{ Acervos } \\
\hline Gêneros & Espécies & Biblioteca A & Biblioteca B & Biblioteca C \\
\hline \multirow[t]{3}{*}{ Acremonium } & A. blochii & 0 & 0 & $1 \%$ \\
\hline & A. kilienses & 0 & 0 & $1 \%$ \\
\hline & Acremonium sp. & $2 \%$ & $2 \%$ & $4 \%$ \\
\hline Alternaria & Alternaria sp. & $2 \%$ & $3 \%$ & $3 \%$ \\
\hline \multirow[t]{9}{*}{ Aspergillus } & Aspergillus alliaceus & 0 & $1 \%$ & 0 \\
\hline & Aspergillus flavus & $19 \%$ & $15 \%$ & $15 \%$ \\
\hline & Aspergillus fumigatus & 0 & $2 \%$ & $2 \%$ \\
\hline & Aspergillus granulosus & 0 & $1 \%$ & $1 \%$ \\
\hline & Aspergillus niger & $10 \%$ & $8 \%$ & $6 \%$ \\
\hline & Aspergillus ochraceus & 0 & $2 \%$ & 0 \\
\hline & Aspergillus terréus & $5 \%$ & $3 \%$ & $5 \%$ \\
\hline & Aspergillus tetrazonus & 0 & $1 \%$ & 0 \\
\hline & Aspergillus sp. & $5 \%$ & $4 \%$ & $2 \%$ \\
\hline Bipolaris & & $2 \%$ & $3 \%$ & $3 \%$ \\
\hline Chaetomium & & 0 & $1 \%$ & 0 \\
\hline Chrysonilia & & 0 & $3 \%$ & $3 \%$ \\
\hline Chrysosporium & Chrysosporium boppi & 0 & 0 & $1 \%$ \\
\hline
\end{tabular}




\begin{tabular}{|c|c|c|c|c|}
\hline $\begin{array}{l}\text { BD } \\
\text { Biblioteconomia e } \\
\end{array}$ & $\begin{array}{l}\text { Revista Digital de } \\
\text { cia da Informação }\end{array}$ & icamp.br/ & dbci & $\begin{array}{l}\text { ARTIGO DE } \\
\text { PESQUISA }\end{array}$ \\
\hline & Chrysosporium inops & $2 \%$ & 0 & $2 \%$ \\
\hline & Chrysosporium sp. & 0 & 0 & $1 \%$ \\
\hline \multirow[t]{2}{*}{ Cladosporium } & $\begin{array}{l}\text { Cladosporium } \\
\text { sphaerospermum }\end{array}$ & 0 & $1 \%$ & 0 \\
\hline & Cladosporium sp. & $8 \%$ & $6 \%$ & $5 \%$ \\
\hline \multicolumn{2}{|l|}{ Cylindrocarpon } & 0 & $1 \%$ & 0 \\
\hline \multicolumn{2}{|l|}{ Cunninghamella } & 0 & 0 & $2 \%$ \\
\hline \multicolumn{2}{|l|}{ Curvularia } & 0 & $3 \%$ & $2 \%$ \\
\hline \multicolumn{2}{|l|}{ Exophiala } & $2 \%$ & $4 \%$ & $2 \%$ \\
\hline \multirow[t]{2}{*}{ Exserohilum } & Exserohilum rostratum & 0 & 0 & $1 \%$ \\
\hline & Exserohilum sp. & 0 & 0 & $2 \%$ \\
\hline \multirow[t]{5}{*}{ Fusarium } & Fusarium napiforme & $1 \%$ & 0 & 0 \\
\hline & Fusarium oxysporum & $1 \%$ & 0 & 0 \\
\hline & Fusarium poliferatum & 0 & 0 & $1 \%$ \\
\hline & Fusarium soloni & 0 & 0 & $1 \%$ \\
\hline & Fusarium sp. & $3 \%$ & $5 \%$ & $3 \%$ \\
\hline \multicolumn{2}{|l|}{ Isaria } & 0 & $1 \%$ & 0 \\
\hline Lecythophora & Lecythophora mutabilis & $1 \%$ & 0 & 0 \\
\hline Mortierella & Mortierella polycephala & $1 \%$ & 0 & 0 \\
\hline \multicolumn{2}{|l|}{ Mucor } & $4 \%$ & 0 & $5 \%$ \\
\hline \multicolumn{2}{|l|}{ Nigrospora } & $2 \%$ & $1 \%$ & $2 \%$ \\
\hline \multicolumn{2}{|l|}{ Ochroconis } & 0 & 0 & $1 \%$ \\
\hline \multicolumn{2}{|l|}{ Paecilomyces } & $2 \%$ & $1 \%$ & $2 \%$ \\
\hline \multicolumn{2}{|l|}{ Penicillium } & $20 \%$ & $18 \%$ & $10 \%$ \\
\hline \multicolumn{2}{|l|}{ Phaeotrichoconis } & 0 & 0 & $1 \%$ \\
\hline \multicolumn{2}{|l|}{ Phialemonium } & 0 & 0 & $1 \%$ \\
\hline \multicolumn{2}{|l|}{ Phialophora } & 0 & 0 & $1 \%$ \\
\hline \multicolumn{2}{|l|}{ Rhizopus } & 0 & 0 & $2 \%$ \\
\hline Scopulariopsis & & $3 \%$ & $2 \%$ & $2 \%$ \\
\hline Scytalidium & & $2 \%$ & $3 \%$ & $1 \%$ \\
\hline Sterella & & 0 & 0 & $1 \%$ \\
\hline Trichoderma & Trichoderma viride & 0 & 0 & $1 \%$ \\
\hline & Trichoderma sp. & $3 \%$ & $5 \%$ & $1 \%$ \\
\hline
\end{tabular}

Para a análise mais apurada sobre a diversidade dos achados micológicos, foram construídos dendogramas cujos itens com maior correlação (normalmente positiva e direta) foram agrupados, considerando a distância de conexão completa (a maior distância entre os termos), já que foi constatada forte similaridade entre os elementos de cada uma das bibliotecas (figura 1). 

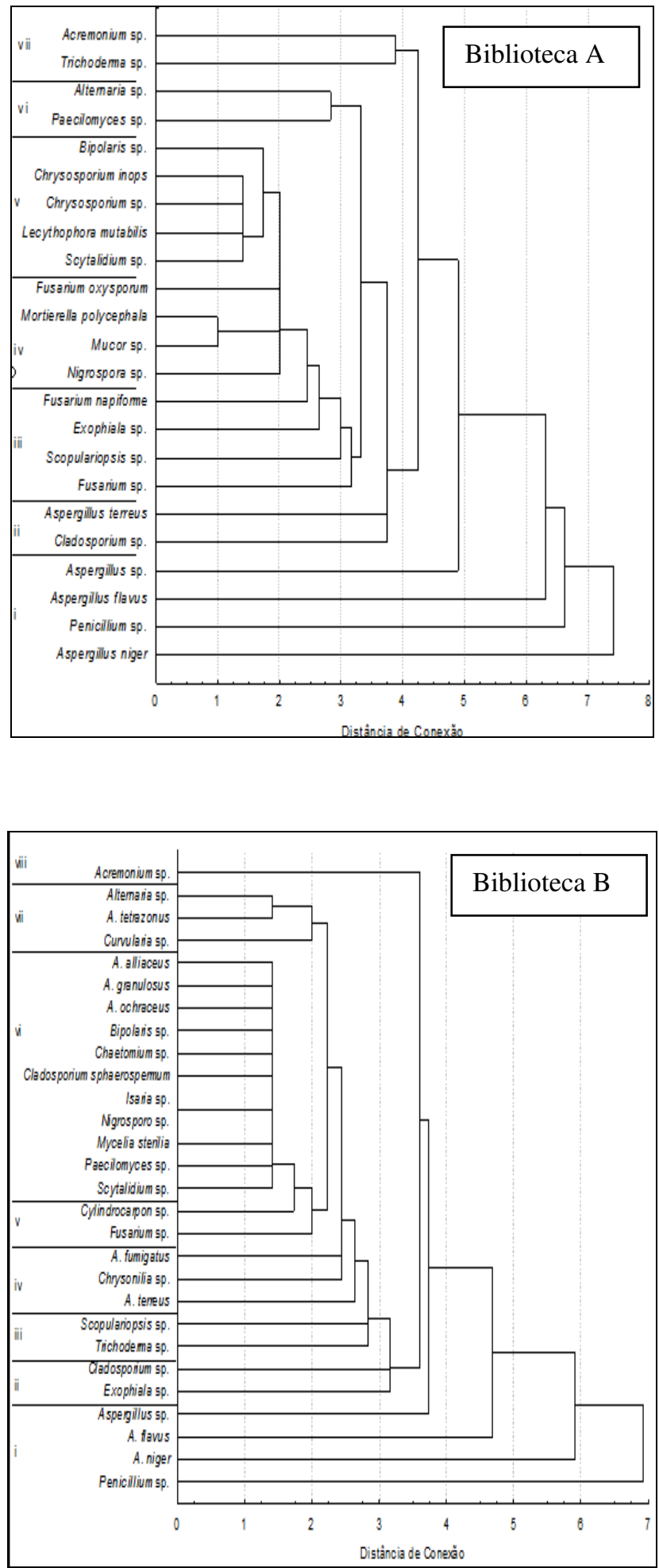


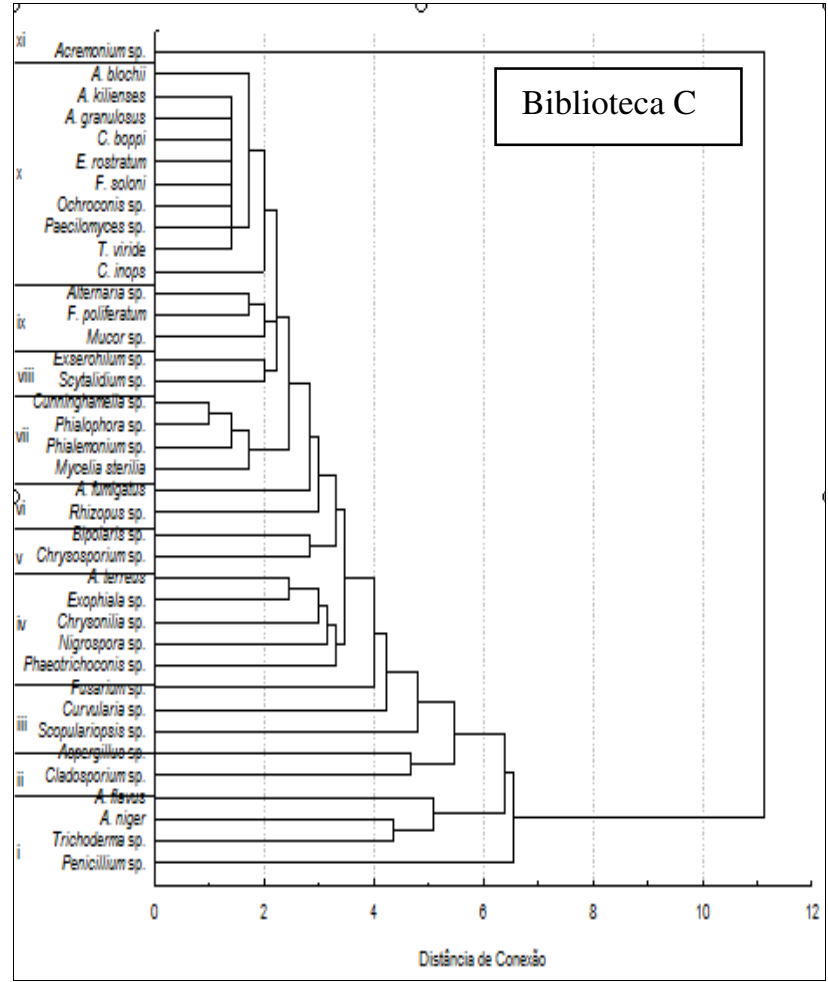

FIGURA 1 - Dendogramas da ocorrência fúngica nos acervos das bibliotecas A, B e C. No eixo y a diversidade de gêneros e espécies fúngicas e no eixo $\mathrm{x}$ a distância de conexão entre os agrupamentos.

Fonte: Próprios autores.

No acervo das bibliotecas A e B Aspergillus sp., Aspergillus flavus, Apergillus niger e Penicillium sp formaram o primeiro agrupamento, e assim sucessivamente. Logo, quando um desses fungos ocorre os outros três também podem estar presentes nas obras analisadas. $\mathrm{Na}$ Biblioteca C percebeu-se forte correlação entre o Aspergillus niger, Trichoderma sp. e Penicillium sp., representando o primeiro agrupamento.

Frente à organização em grupos, a biblioteca $\mathrm{C}$ apesar da menor contagem de colônias (2.978) apresentou o maior número de agrupamentos (11), seguido da biblioteca B, com 08 agrupamentos e da biblioteca A com 07 agrupamentos. Com a análise dos dados, observou-se que não existe relação entre quantidade de unidades formadoras de colônias e a organização em agrupamentos $(\mathrm{p} \geq 0,05)$.

A diversidade fúngica observada coaduna-se aos trabalhos de Gallo (1993) e Rosa e colaboradores (2008), que mostraram que os representantes fúngicos mais frequentemente associados a livros e documentos tratavam-se dos Deuteromycetes pertencentes aos gêneros Aspergillus, Penicillium, Trichoderma, Stachybooys, Stemphylium, Alternaria, Myrothecium seguidos pelos Zygomycetes (Rhizopus e Mucor) e Ascomycetes (Chaetomium). 


\section{CONSIDERAÇÕES FINAIS}

Destaca-se a significativa positividade e diversidade do espectro fúngico encontrado nos acervos bibliográficos analisados, bem como a existência de agrupamentos com forte correlação, em especial com os hialomicetos Aspergillus e Penicillium.

A despeito da imensurável importância das bibliotecas públicas para o patrimônio cultural da humanidade, nem sempre tais instituições dispõem de corpo técnico habilitado para sua correta manutenção, nem dotações orçamentárias compatíveis, sendo necessário a continuidade de estudos correlacionando datações das obras, tipos de climatização do ambiente, entre outros, sendo fato que muito ainda precisa ser feito frente à qualidade dos acervos de bibliotecas.

Nesse ínterim, a pesquisa ofereceu aos gestores das bibliotecas, elementos para aprofundar seus conhecimentos na área de preservação e conservação de obras literárias de inestimável valor, norteando a adoção de normas mínimas no trato com as unidades documentais e o espaço físico das bibliotecas, além de contribuir com a compilação de dados epidemiológicos das ocorrências fúngicas que podem repercutir ainda na conservação da saúde de seus usuários.

\section{REFERÊNCIAS}

AAS, K. The allergic child. In: C. Thomas (Ed.), Chest. Springfield, Illinois. 1972. ALEXOPOULOS, C. J.; MIMS, C. W.; BLAKWEL, L. Introductory mycology. 4. ed. New York: John Wiley e Sons, 1996. 869 p.

ARABIDIAN, L. V.; SAAD, D. S. Avaliação da contaminação microbiana e de parâmetros ambientais - temperatura, ventilação e umidade - na Biblioteca Central da Universidade Federal de Santa Maria/RS: acervos da Coleção Teses e Coletânea UFSM. Revista da Faculdade de Biblioteconomia e Comunicação da UFRGS, v. 20, n.2 - Jul./Dez. 2014.

BRASIL. MINISTÉRIO DA SAÚDE. Orientação técnica sobre padrões referenciais de qualidade do ar interior em ambientes climatizados artificialmente de uso público e coletivo. Resolução $\mathbf{n}^{\mathbf{0}}$ 176, 24 de outubro de 2000. Brasília: Centro de Documentação do Ministério da Saúde, 2000.

BRICKUS, L. R.; AQUINO NETO, F. R. A Qualidade do Ar de Interiores e a Química. Química Nova, v. 22, n. 1, p. 10. 1999.

BORTOLETO, M. E.; MACHADO, R. R.; COUTINHO, E. Contaminação fúngica do acervo da biblioteca de Manguinhos da Fundação Oswaldo Cruz. Ações desenvolvidas para sua solução. Revista Eletrônica Biblioteconomia Florianópolis, v. 14, p. 1-10. 2002.

GALLO, F.; GALLO, P. Esperienze nel campo della disinfezione e disinfestazione con ossido di etilene. Seminario di studi su tutela dei documenti di archivio, disinfezione e disinfestazione con ossido di etilene. Local: Roma, 1988. 
GAMBALE, W.; CROCE, J.; COSTA-MANSO, R.; CROCE, M.; SALES, M. Library fungi at University of São Paulo and their relationship with respiratory allergies. Journal of Investigative Allergology and Clinical Immunology, v. 3, p. 45-50. 1993.

HOOG, G. S. de; GUARRO, J.; GENÉ, J.; FIGUEIRAS, M. J. Atlas of Clinical Fungi. 2. ed. Baarn/Delft: Centraalbureau voor Schinmelculture/Universitat Rovira i Virgilli, 2000. $2.108 \mathrm{p}$.

HUANG, C-Y.; LEE, C-C.; MA, Y.; SU, H-J. The seasonal distribution of bioaerosols in municipal landfill sites: a 3-yr study. Atmospheric Environment, v. 36, p. 4385-4395. 2002.

HUSSON, F.; JOSSE, J.; LÊ, S.; MAZET, J. (2009). FactoMineR: Factor Analysis and Data Mining with R. R Package Version 1.12. Disponível em: <http://www.CRAN.Rproject.org/package=FactoMineR>. Acesso em: $15 \mathrm{dez} 2009$.

KONEMAN, E.; ALLEN, S.; JANDA, W.; SCHRECKENBERGER, P.; WINN Jr. W. Diagnóstico microbiológico: texto e atlas colorido. 5. ed. Rio de Janeiro: MEDSI, 2001. 2067 p.

LACAZ, C. S.; PORTO, C. E.; MARTINS, J. E.; HEINS-VACCARI, E. M.; MELO, T. Fungos e Alergia. In: Tratado de Micologia Médica Lacaz. São Paulo: Sarvier, 2002. cap. 35 , p. $810-828$.

LI, C-S.; KUO, Y-M. Airbone characterization of fungi indoors and outdoors. J. Aerosol Sci, v. 23, p. 667-670. 1992.

MAIN, C. Aerobiological, ecological and health linkages. Environment International, v. 29 , n. 2/3, p. 347-349. 2003.

MADIGAN, M.T.; MARTINKO, J.M.; PARKERT, J. Microbiologia de Brock. 12 ed. Ed. Prentice Hall. 2010.

MENEZES, E. A.; ALCANFOR, A. C.; CUNHA, F. A. Airborne fungi in the periodics room of the library of health science of the University Federal of Ceará. Revista Brasileira de Análises Clínicas, v. 38, p. 155-158. 2006.

MOBIN, M. Myxomycetes e fungos micófilos ocorrentes em palmeiras no Parque Nacional de Sete Cidades (Piripiri - Piauí - Brasil). 116 f. Dissertação de Mestrado. Universidade Federal de Pernambuco, Recife, 1997.

PANTOJA, L. D. M; COUTO, M. S.; PAIXÃO, G. C. Diversidade de Bioaerossóis presentes em ambientes urbanizados e preservados de um Campus universitário. Biológico, v.69, p.4147, 2007.

PANTOJA, L. D. M.; RIZZO, R. S.; CARVALHO, B. S.; FERREIRA, V. C.; GALAS, K. S.; FONSECA, F. R. M.; PAIXÃO, B. C. Constituição da micobiota aérea de bibliotecas 
públicas no município de Fortaleza, Estado do Ceará, Brasil. Encontros Bibli: revista eletrônica de biblioteconomia e ciência da informação, v. 17, n. 34, p.31-41. 2012.

PASTUSZKA, J.; PAW, K. T.; LIS, D.; WLAZLO, A.; ULFIG, K. Bacterial and fungal aerosol in indoor environment in Upper Silesia, Poland. Atmospheric Environment, v. 34, p. 3833-3842. 2000.

PEI-CHIN, W.; HUEY-JEN, S.; CHIA-YIN, L. Characteristics of indoor and outdoor airbone fungi at suburban and urban homes in two seasons. The Science of the Total Environment, v. 253, p. 111-118. 2000.

PIACENTI, M. R. Conservação e preservação de fotografias impressas em papel: um estudo de caso na Câmara dos Deputados. Monografia (Bacharelado em Biblioteconomia) Universidade de Brasília, Faculdade de Ciência da Informação, Curso de Biblioteconomia, 2014.

RÊGO, R. S. de M.; MAGALHÂES, K.; MELO, M.; SILVEIRA, N. S. S. Ocorrência de Aspergillus sp em pacientes com sinusite crônica. In: Programa oficial e Anais do $4^{\mathbf{0}}$ Congresso Brasileiro de Micologia, Universidade Federal de Ouro Preto, Ouro Preto, p.135, 2004.

REPONEN, T.; WILLEKE, K.; ULEVICIUS, V.; REPONEN, A.; GRINSHPUN, S. Effect of relative humidity on the aerodynamic diameter and respiratory deposition of fungal spores. Atmospheric Environment, v. 30, p. 3967-3974. 1996.

ROSA, H.; LEMOS, J. de A.; COSTA, C. R.; SILVA, M. do R.; FERNANDES, O. de F. Ocorrência de fungos filamentosos em acervo da faculdade de Medicina da Universidade Federal de Goiás. Revista de Patologia Tropical, v. 37, p. 65-69. 2008.

R CORE TEAM. R: A language and environment for statistical computing. R Foundation for Statistical Computing, Vienna, Austria. ISBN 3-900051-07-0, URL http://www.Rproject.org/. 2012.

SIDRIM, J. J. C.; ROCHA, M. F. G. Micologia Médica à Luz de Autores Contemporâneos. Rio de Janeiro: Guanabara Koogan, 2004.

VALENTIN, J. L. Ecologia numérica: uma introdução à análise multivariada de dados ecológicos. Rio de Janeiro: Interciência Ltda. 2000. 117 p.

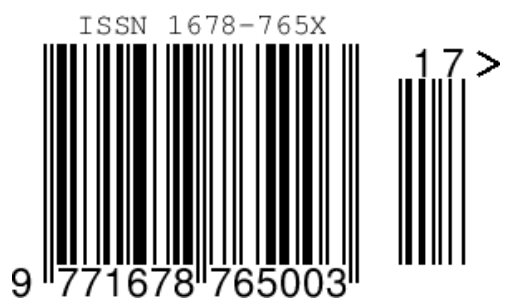


PAIXÃO, Germana Costa et al. Ocorrência fúngica em acervos bibliográficos do município de Fortaleza, Ceará, Brasil. RDBCI: Revista Digital de Biblioteconomia e Ciência da Informação, Campinas, SP, v. 14, n. 1, p. 180-191, fev. 2016. ISSN 1678-765X. Disponível em: <http://periodicos.sbu.unicamp.br/ojs/index.php/rdbci/article/view/8640649>. Acesso em: 01 fev. 2016. doi:http://dx.doi.org/10.20396/rdbci.v14i1.8640649. 